Research Article

\title{
5-Aminosalicylic Acid Prevents Disease Behavior Progression and Intestinal Resection in Colonic and Ileocolonic Crohn's Disease Patients: A Retrospective Study
}

\author{
Jian Wan $\mathbb{D}^{1},{ }^{1}$ Xuan Wang $\mathbb{D}^{2},{ }^{2}$ Yujie Zhang, ${ }^{1,3}$ Xianmin Xue, ${ }^{1}$ Yani Li, ${ }^{1}$ Zhenzhen Liu, ${ }^{1}$

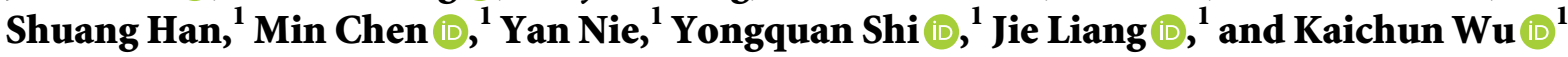 \\ ${ }^{1}$ State Key Laboratory of Cancer Biology, \\ National Clinical Research Center for Digestive Diseases and Xijing Hospital of Digestive Diseases, \\ Fourth Military Medical University, Xi'an, Shaanxi Province, China \\ ${ }^{2}$ Department of Neurology, Xijing Hospital, Fourth Military Medical University, Xi'an, Shaanxi Province, China \\ ${ }^{3}$ Department of Histology and Embryology, School of Basic Medicine, Xi'an Medical University, Xi'an, China
}

Correspondence should be addressed to Jie Liang; liangjie@fmmu.edu.cn and Kaichun Wu; kaicwu@fmmu.edu.cn

Received 22 May 2021; Accepted 31 July 2021; Published 10 August 2021

Academic Editor: Rafał Filip

Copyright $\odot 2021$ Jian Wan et al. This is an open access article distributed under the Creative Commons Attribution License, which permits unrestricted use, distribution, and reproduction in any medium, provided the original work is properly cited.

\begin{abstract}
Background and Aims. The efficacy of 5-aminosalicylic acid (5-ASA) in the long-term outcome of Crohn's disease (CD) patients was uncertain. This study aimed to evaluate the efficacy of the 5-ASA in preventing disease behavior progression and intestinal resection in CD patients. Methods. CD patients were prospectively enrolled from January 2008 to September 2019 in Xijing Hospital. Disease behavior progression was defined as the development of stricturing (B2) or penetrating disease (B3) in patients with nonstricturing/nonpenetrating disease (B1) at diagnosis. Cox regression analyses were used to investigate the associations between disease location progression, disease behavior progression, and intestinal resection and multiple covariates. Results. In total, $122 \mathrm{CD}$ patients were followed up for 4.3 years. At the time of diagnosis, disease location was ileal in $19.7 \%$ (24/122), colonic in 41.0\% (50/122), and ileocolonic in 39.3\% (48/122). A total of 87 (71.3\%) patients had B1 at diagnosis. The disease behavior progression and intestinal resection rates were $42.5 \%(37 / 87)$ and $29.5 \%(36 / 122)$. The use of 5-ASA reduced the risk of disease behavior progression (HR 0.30, 95\% CI 0.14-0.61, $P=0.001)$ and intestinal resection (HR 0.33, 95\% CI $0.17-0.90, P=0.027$ ) in colonic and ileocolonic CD patients. Patients who presented with ileal disease at diagnosis did not have the same protective effects when taking 5-ASA $(P>0.05)$. Conclusions. The use of 5-ASA could improve the long-term outcome of CD patients with colon involvement. The result emphasized the importance of early use of 5-ASA in the daily management of colonic involved CD.
\end{abstract}

\section{Introduction}

Crohn's disease $(\mathrm{CD})$ is an inflammatory bowel disease (IBD) that may involve the whole gastrointestinal tract [1]. The incidence of CD has increased sharply in China $[1,2]$. $\mathrm{CD}$ is a progressive and dynamic disease that leads to bowel damage and disability [3].

Population-based studies demonstrate that disease location is relatively stable in $\mathrm{CD}$ patients and is presented with ileal, ileocolonic, or colonic disease in about one-third each [3]. Only about $6.5 \%-13.5 \%$ of the patients experience a change in disease location [4]. A multicenter prospective disease registry study in China demonstrates that more than half of the CD patients were presented with ileocolonic disease at diagnosis and the rates of ileal and colonic disease were $27.8 \%$ and $14.4 \%$ [5]. Furthermore, about $56 \%-81 \%$ of $\mathrm{CD}$ patients have inflammatory disease behavior at diagnosis, whereas about $5 \%-25 \%$ present with stricturing or penetrating disease behavior $[3,4]$. Cumulative risk of developing stricturing or penetrating disease among those patients with inflammatory behavior is $18 \%$ at 7 years [6] and $51 \%$ at 20 years [7] after diagnosis. Risk factors 
associated with developing stricturing or penetrating disease include young age at diagnosis [8], change in disease location [6], ileal/ileocolonic involvement, and penetrating or stricturing disease phenotype [9]. However, these factors have poor precision as predictors and are not widely accepted as accurate predictors [1]. There is a high rate of surgery in the CD patients. The cumulative risk of surgery 10 years after diagnosis was about $40-55 \%$ based on studies conducted in Western countries [3,4].

Current strategies of CD treatment usually focus on the induction and maintenance of remission, prevention of complications and disease progression, and reducing the risk of surgery [1]. In the past, the initial treatment for $\mathrm{CD}$ patients was always the use of 5-aminosalicylic acid (5-ASA). Although the recent European Crohn's and Colitis Organization (ECCO) consensus has suggested against the use of 5-ASA for induction and maintenance of remission of Crohn's disease [10], 5-ASA is still widely used for the treatment of CD, especially in patients who presented with colonic or ileocolonic disease location, inflammatory disease behavior, and mild disease activity [11-13]. In a Swiss IBD Cohort, $59 \%$ of the CD patients were treated with 5-ASA before [13]. Forty-seven percent of children and adolescent CD patients were treated with 5-ASA during their disease course [14]. In China, about $58 \%-73 \%$ of the CD patients have been treated with 5-ASA before, especially 35\% using 5ASA as the only medicine [15-17]. As the most widely used medicine for CD [18], efficacy and suitable subtype of CD for the use of 5-ASA would be of importance for investigation. Many studies focused on the efficacy of 5-ASA in CD patients for the induction remission, prevention of relapse, and maintenance of surgically induced remission [19] but the results were conflicting and uncertain [20]. Some studies showed that sulfasalazine might have efficacy in remission induction in colonic CD [21, 22]. Since the isolated colonic $\mathrm{CD}$ was thought to be quite different from $\mathrm{CD}$ with small intestinal involvement [23], it can be speculated that 5-ASA might just only have efficacy in colonic involved CD patients, but not in all the CD patients. In addition, few studies focused on the efficacy of 5-ASA in the long-term outcome of CD patients, such as the disease location and behavior progression and intestinal resection.

In this study, we aimed to evaluate the use of the 5-ASA in reducing the risk of disease progression and surgery in CD patients, especially in the colon involved patients.

\section{Methods}

2.1. Study Population and the Endpoint of Study. The CD patients who were diagnosed in the Department of Digestive Disease, Xijing Hospital, and followed up at the specialist clinic for IBD from January 2008 to September 2019 were prospectively recruited into a database. The staffs of the IBD clinic include experienced gastroenterologists, gastrointestinal specialist pathologists, radiologists, nurses, specialist surgeons, and nutritionists. CD was diagnosed based on the medical history and clinical manifestations, combined with the endoscopic and histological findings according to the Chinese consensus on diagnosis and treatment in IBD, which agrees with the ECCO consensus [24, 25]. For all patients included in our study, we required a confirmed diagnosis of CD and a follow-up of at least 6 months after the diagnosis. Patients with uncertain diagnosis or incomplete data were excluded.

The primary outcome of our study was the change of behavior. The secondary outcomes were the change of location and intestinal resection. The endpoint of each outcome was defined as the data of change in location, behavior, and intestinal resection during surveillance. If each outcome of the patient did not develop, the patients were censored at the date of the latest available colonoscopy up to September 2019.

2.2. Classifications and Definitions. In our study, all the CD patients were categorized according to the Montreal classification [26] both at diagnosis and throughout the follow-up period. Age at diagnosis (A) was categorized as three levels: A1 (<17 years), A2 (17-40 years), and A3 ( $>40$ years). Disease location $(L)$ included ileal $(L 1)$, colonic (L2), ileocolonic (L3), and upper gastrointestinal diseases (L4). Change in disease behavior $(B)$ in our study was defined as the development of $B 2$ (stricturing) or $B 3$ (penetrating) in patients with $B 1$ (nonstricturing, nonpenetrating) at diagnosis.

2.3. Treatment and Follow-Up Policy. All the CD patients were followed up regularly at the specialist clinic of IBD every 1 to 6 months based on the patients' conditions. Medication was defined as first exposition to a certain treatment. Treatment included 5-ASA (oral or topical), corticosteroid (not included topical corticosteroids), immunomodulators, biologics (infliximab or adalimumab), and intestinal resection. Intestinal resection was defined as the resection of a part of the bowel because of uncontrolled intestinal inflammation or a CD-related complication.

2.4. Clinical Data Collection. Medical histories of the study patients were reviewed and clinical data were collected, which include age, age at onset, gender, disease duration, appendicectomy history, Crohn's Disease Activity Index (CDAI), erythrocyte sedimentation rate (ESR), smoking, clinical features, disease location and behavior and perianal disease when diagnosed and during follow-up, each endoscopy result, and treatment. Smoking status was recorded at the time of diagnosis. Current smoking was defined as patients who had smoked at least 6 months before diagnosis, while former smoking was defined as stopping smoking for at least 6 months. The endoscopy reports of every study patient and the associated images were reviewed by experienced endoscopist (Y.Z.). Any disagreements with original record were solved by discussion with another experienced endoscopist (K.W.).

2.5. Ethical Considerations. The study was approved by the ethical committee of Xijing Hospital affiliated to the Fourth Military Medical University in Xi'an, China. All of the 
patients or their legal representatives signed the informed consent form at the time of enrolling in the cohort.

2.6. Statistical Methods. The data were analyzed using the SPSS 19.0 (IBM, Armonk, NY, USA) computer software for Windows. Quantitative variables were summarized as median and interquartile range (IQR). Categorical variables were expressed as frequency and percentage (\%). Two-tailed $t$-test or Mann-Whitney test was used to compare the continuous variables for data and the chi-square and Fisher's exact test were used to compare the frequencies of categorical variables, as appropriate. Cox regression was used to select risk factors associated with the occurrence of each outcome. The factors which were significant $(P<0.10)$ at the univariate analysis were included in the final cox proportional hazards model. We used hazard ratios (HRs) with 95\% confidence intervals (CIs) to quantify the association of the factors with each outcome.

\section{Results}

A total of $141 \mathrm{CD}$ patients were reviewed and 19 patients had incomplete data or no follow-up data. As a result, 122 patients were enrolled in our study. The median follow-up time was 4.3 years (IQR 2.5-7.1 years). Patient characteristics are presented in Table 1. A total of 92 patients had received 5ASA. No significance was found between patients treated with and without 5-ASA.

3.1. Change in Disease Behavior. Of 87 patients with $\mathrm{B} 1$ at diagnosis, $31.0 \%(27 / 87)$ of the patients progressed to $B 2$, and $11.5 \%(10 / 87)$ of the patients progressed to $B 3$. The distribution of disease behavior over the first 7 years of disease is shown in Figure 1. The median time of change in disease behavior in patients who presented with $B 1$ at diagnosis was 30.5 months (IQR 12.8-60.3 months). Of patients with $B 2$ at diagnosis, $13.3 \%(4 / 30)$ of the patients progressed to $B 3$. The cumulative risk of change in disease behavior in patients who presented with $B 1$ at diagnosis is shown in Figure 2. Multivariate analysis showed that factors associated with change in disease behavior in patients with $B 1$ at diagnosis were the use of 5-ASA (HR 0.29, 95\% CI $0.14-0.58, P=0.001$ ) and appendicectomy (HR 0.32, 95\% CI $0.12-0.82, P=0.018$ ) (Table 2). Biologics treatment was of significance in the univariate analysis (HR 0.43, 95\% CI $0.20-0.95, P=0.037)$ and lost significance in the multivariate analysis (HR $0.47,95 \%$ CI $0.21-1.08, P=0.075$ ) (Table 2).

Subgroup analysis showed that the use of 5-ASA (HR $0.30,95 \%$ CI $0.14-0.61, P=0.001)$ and appendicectomy (HR $0.30,95 \%$ CI $0.11-0.78, P=0.013)$ was significantly associated with change to $B 2 / B 3$ from $B 1$ in patients with $L 2$ and L3 at diagnosis (Table 2; Figure 3). No factor was significant in the patients presenting with $L 1$ at diagnosis (data not shown, $P>0.05)$.

3.2. Change in Disease Location. Of the $8(8 / 122,6.6 \%)$ patients diagnosed with $L 4$, the numbers of patients coexisting with $L 1, L 2$, and $L 3$ were 2, 3, and 3, respectively.
Changes in disease location were observed in 30 patients (24.6\%) during follow-up. Of the patients with $L 1$ at diagnosis, $25.0 \%(6 / 24)$ changed to $L 3$. Of the patients with $L 2$ at diagnosis, $40.0 \%(20 / 50)$ changed to $L 3$ and $4.0 \%(2 / 50)$ to $L 2+L 4$. Of the patients diagnosed with $L 3$, only $4.2 \%(2 / 48)$ changed to $L 3+L 4$. The cumulative risk of change in disease location in $L 1$ and $L 2$ patients is shown in Figure 2 . A total of 26 patients presented with $L 1$ and $L 2$ at diagnosis changed to L3 during follow-up. The median time of change in disease location in these patients was 29.5 months (IQR 10.0-70.0 months). None of the risk factors was significantly associated with change in disease location (Table S1).

3.3. Intestinal Resections. A total of 36 (29.5\%) patients had intestinal resection in our study. The median time of intestinal resection in all the $122 \mathrm{CD}$ patients was 17.0 months (IQR 5.0-37.8 months). The number of patients who had intestinal resection during the first and second year after diagnosis was $16(44.4 \%)$ and $9(25.0 \%)$. The majority of the 36 patients were $A 2(47.2 \%, 17 / 36)$ at the time of diagnosis, followed by $A 3(27.8 \%, 10 / 36)$ and $A 1(25 \%, 9 / 36)$. Most of the 36 patients presented with $L 2(41.7 \%, 15 / 36)$ and $L 1$ $(30.6 \%, 11 / 36)$ at the time of diagnosis, followed by $L 3$ $(19.4 \%, 7 / 36)$ and $L 4(8.3 \%, 3 / 36)$. For disease behavior at the time of diagnosis, most patients were $B 1(63.9 \%, 23 / 36)$, followed by $B 2(25.0 \%, 9 / 36)$ and $B 3(11.1 \%, 4 / 36)$. The cumulative risk of intestinal resection is shown in Figure 2. Multivariate analysis showed that risk factors associated with intestinal resection were $B 3$ at diagnosis ( $\mathrm{HR} 4.65,95 \% \mathrm{CI}$ $1.24-17.34, P=0.022$ ), the use of 5 -ASA (HR $0.40,95 \% \mathrm{CI}$ $0.20-0.87, P=0.024)$, and the use of immunomodulators (HR 0.29, 95\% CI 0.09-0.88, $P=0.030$ ) (Table 3).

Subgroup analysis was performed according to the disease location at diagnosis. The 5-ASA (HR 0.33, 95\% CI $0.17-0.90, P=0.027$ ) and biologics (HR $0.24,95 \% \mathrm{CI}$ $0.07-0.82, P=0.023)$ were used as protective factors for intestinal resection in patients who presented with $L 2$ and $L 3$ at diagnosis. No factor was significant in the patients presenting with $L 1$ at diagnosis (Table S2).

\section{Discussion}

Our study demonstrated that the use of 5-ASA could reduce the risk of disease behavior progression and intestinal resection in patients with $L 2$ and $L 3$ at diagnosis. A cohort of $122 \mathrm{CD}$ patients was followed up with a median of 4.3 years. About a quarter of the patients had a change in disease location. At diagnosis, more than seventy percent of the patients presented with $B 1$ and $42.5 \%$ of the $B 1$ patients progressed to $B 2$ or $B 3$. About thirty percent of the patients had intestinal resection and most of the resection occurred in the first two years. Patients who presented with B3 at diagnosis were associated with higher rate of intestinal resection, while the use of immunomodulators was a protective factor for intestinal resection. Biologics use decreased the risk of intestinal resection in patients with $L 2$ and $L 3$ at diagnosis. 
TABle 1: Characteristics of Crohn's disease patients.

\begin{tabular}{|c|c|c|c|c|}
\hline & Results $(n=122)$ & Non-5-ASA $(n=30)$ & 5-ASA $(n=92)$ & $P$ value \\
\hline Age, median (IQR) & $33.0(26.0-46.0)$ & $32.5(20.5-40.3)$ & $33.5(28.0-46.0)$ & 0.082 \\
\hline Age at diagnosis, median (IQR) & $27.0(21.0-40.0)$ & $28.0(16.0-39.0)$ & $27.0(22.0-41.8)$ & 0.184 \\
\hline Female, $n(\%)$ & $50(41.0)$ & $12(40.0)$ & $38(41.3)$ & 0.900 \\
\hline Smoking, $n(\%)$ & & & & 0.330 \\
\hline Never & $95(77.9)$ & $25(83.3)$ & $70(76.1)$ & \\
\hline Former & $2(1.6)$ & $1(3.3)$ & $1(1.1)$ & \\
\hline Current & $25(20.5)$ & $4(13.3)$ & $21(22.8)$ & \\
\hline Age at diagnosis, $n(\%)$ & & & & 0.070 \\
\hline$A 1$ & 17 (13.9) & $8(26.7)$ & $9(9.8)$ & \\
\hline$A 2$ & $76(62.3)$ & $17(56.7)$ & $59(64.1)$ & \\
\hline$A 3$ & $29(23.8)$ & $5(16.7)$ & $24(26.1)$ & \\
\hline Location at diagnosis, $n(\%)$ & & & & 0.753 \\
\hline$L 1$ & $24(19.7)$ & $5(16.7)$ & $19(20.7)$ & \\
\hline$L 2$ & $50(41.0)$ & $14(46.7)$ & $36(39.1)$ & \\
\hline$L 3$ & $48(39.3)$ & $11(36.7)$ & $37(40.2)$ & \\
\hline L4 involvement & $8(6.6)$ & $3(10.0)$ & $2(2.2)$ & 0.095 \\
\hline Behaviour at diagnosis, $n(\%)$ & & & & 0.403 \\
\hline$B 1$ & $87(71.3)$ & $23(76.7)$ & $64(69.6)$ & \\
\hline$B 2$ & $30(24.6)$ & $5(16.7)$ & $25(27.2)$ & \\
\hline$B 3$ & $5(4.1)$ & $2(6.7)$ & $3(3.3)$ & \\
\hline Perianal disease at diagnosis, $n(\%)$ & $5(4.1)$ & $1(3.3)$ & $4(4.3)$ & 1.000 \\
\hline Appendicectomy, $n(\%)$ & $31(25.4)$ & $8(26.7)$ & $23(25.0)$ & 0.856 \\
\hline Elevated ESR at diagnosis, $n(\%)$ & $73(59.8)$ & $19(63.3)$ & $54(58.7)$ & 0.653 \\
\hline CDAI at diagnosis, median (IQR) & $171.0(102.0-259.5)$ & $161.0(100.0-342.0)$ & $173.0(102.0-246.0)$ & 0.562 \\
\hline \multicolumn{5}{|c|}{ Cumulative exposures for treatment, $n(\%)$} \\
\hline 5-Aminosalicylic acid & $92(75.4)$ & - & - & - \\
\hline Corticosteroid & $68(55.7)$ & $15(50.0)$ & $53(57.6)$ & 0.466 \\
\hline Immunomodulators & $64(52.5)$ & $13(43.3)$ & $51(55.4)$ & 0.249 \\
\hline Biologics & $70(57.4)$ & $18(60.0)$ & $52(56.5)$ & 0.738 \\
\hline
\end{tabular}

IQR, interquartile range; ESR, erythrocyte sedimentation rate; CDAI: Crohn's disease activity index; 5-ASA, 5-aminosalicylic acid.

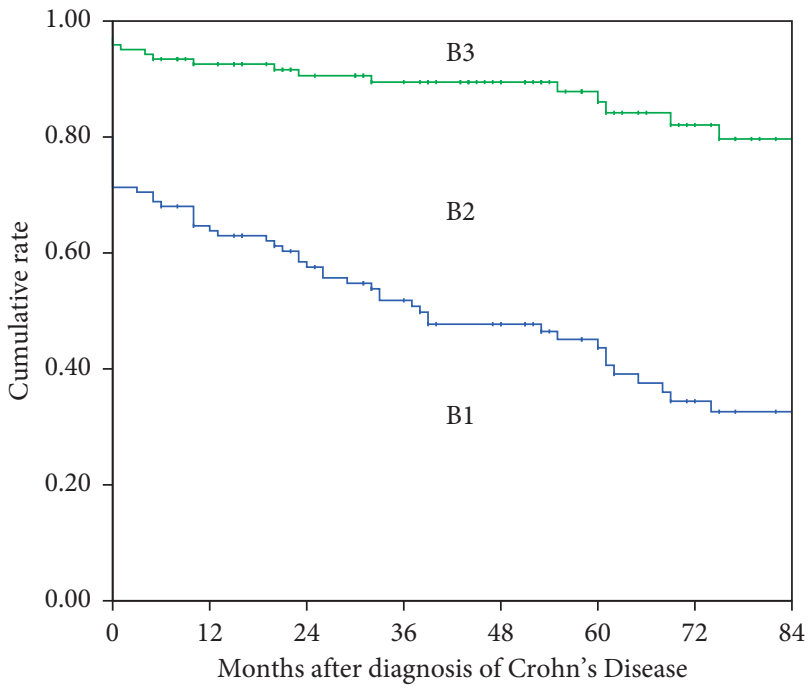

FIGURE 1: The distribution of disease behavior over the first 7 years of disease. $B 1$, nonstricturing, nonpenetrating disease; $B 2$, stricturing disease; $B 3$, penetrating disease.

Disease location at diagnosis was most presented at colitis and ileocolitis, which was similar with a Europe-wide population-based study [27]. However, in an Asia-Pacific region population-based study, about half of the $\mathrm{CD}$ patients

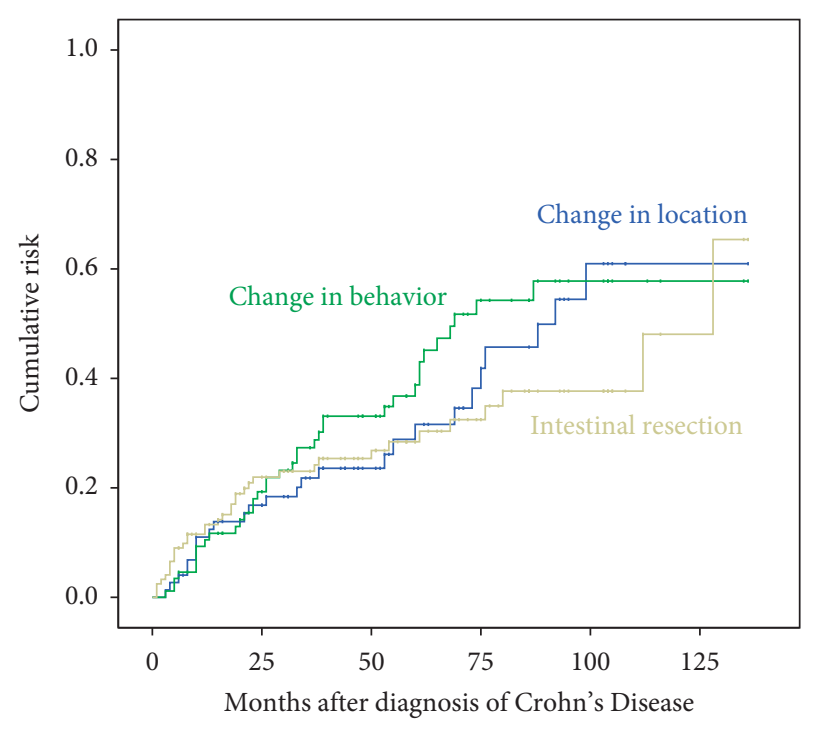

FIgure 2: Cumulative risk of change in disease location, disease behavior, and intestinal resection.

presented with ileocolitis at diagnosis, and the other two types of location accounted for a quarter each [28]. Previous study had shown that the disease location remained stable $[1,3,29]$. Only $6.5-13.5 \%$ of the CD patients had 
TABLE 2: Factors associated with change in disease behavior

\begin{tabular}{|c|c|c|c|c|c|c|c|c|}
\hline & \multicolumn{4}{|c|}{ All B1 patients $(n=87)$} & \multicolumn{4}{|c|}{$(L 2+\mathrm{L} 3)$ in B1 patients $(n=71)$} \\
\hline & \multicolumn{2}{|r|}{ Univariate } & \multicolumn{2}{|r|}{ Multivariate } & \multicolumn{2}{|r|}{ Univariate } & \multicolumn{2}{|c|}{ Multivariate } \\
\hline & $P$ & HR $(95 \% \mathrm{CI})$ & $P$ & HR $(95 \% \mathrm{CI})$ & $P$ & HR $(95 \% \mathrm{CI})$ & $P$ & HR $(95 \% \mathrm{CI})$ \\
\hline Female & 0.387 & $0.75(0.39-1.45)$ & NS & & 0.418 & $0.73(0.35-1.55)$ & NS & \\
\hline \multicolumn{9}{|l|}{ Smoking } \\
\hline Former vs. never & 0.974 & NA & NS & & 0.984 & NA & NA & \\
\hline Current vs. never & 0.736 & $1.13(0.55-2.34)$ & NS & & 0.796 & $0.90(0.40-2.01)$ & NS & \\
\hline \multicolumn{9}{|l|}{ Age at diagnosis } \\
\hline$A 2$ vs. $A 1$ & 0.246 & $2.03(0.61-6.72)$ & NS & & 0.224 & $2.45(0.58-10.37)$ & NS & \\
\hline$A 3$ vs. $A 1$ & 0.199 & $2.36(0.64-8.74)$ & NS & & 0.284 & $2.46(0.47-12.74)$ & NS & \\
\hline \multicolumn{9}{|l|}{ Location at diagnosis } \\
\hline$L 2$ vs. $L 1$ & 0.360 & $1.53(0.61-3.82)$ & NS & & NA & & NA & \\
\hline$L 3$ vs. $L 1$ & 0.931 & $1.04(0.39-2.80)$ & NS & & NA & & NA & \\
\hline L4 involvement & 0.866 & $1.08(0.42-2.78)$ & NS & & 0.894 & $0.93(0.33-2.66)$ & NS & \\
\hline Perianal disease & 0.398 & $1.68(0.51-5.57)$ & NS & & 0.241 & $2.07(0.62-6.96)$ & NS & \\
\hline Appendicectomy & 0.007 & $0.27(0.11-0.70)$ & 0.018 & $0.32(0.12-0.82)$ & 0.022 & $0.33(0.13-0.85)$ & 0.013 & $0.30(0.11-0.78)$ \\
\hline Elevated ESR at diagnosis & 0.559 & $1.22(0.63-2.33)$ & NS & & 0.172 & $1.65(0.80-3.38)$ & NS & \\
\hline CDAI at diagnosis & 0.180 & $1.00(1.00-1.01)$ & NS & & 0.115 & $1.00(1.00-1.01)$ & NS & \\
\hline 5-Aminosalicylic acid & $\leq 0.001$ & $0.29(0.15-0.55)$ & 0.001 & $0.29(0.14-0.58)$ & 0.002 & $0.32(0.16-0.66)$ & 0.001 & $0.30(0.14-0.61)$ \\
\hline Corticosteroid & 0.140 & $0.60(0.31-1.18)$ & NS & & 0.633 & $0.84(0.42-1.71)$ & NS & \\
\hline Immunomodulators & 0.085 & $0.52(0.25-1.10)$ & 0.854 & $0.93(0.41-2.11)$ & 0.179 & $0.59(0.27-1.28)$ & NS & \\
\hline Biologics & 0.037 & $0.43(0.20-0.95)$ & 0.075 & $0.47(0.21-1.08)$ & 0.130 & $0.54(0.24-1.20)$ & NS & \\
\hline
\end{tabular}

HR, hazard ratio; ESR, erythrocyte sedimentation rate; NA, not available; NS, not significance $(P>0.10)$ in the univariate Cox regression; CDAI: Crohn's disease activity index.

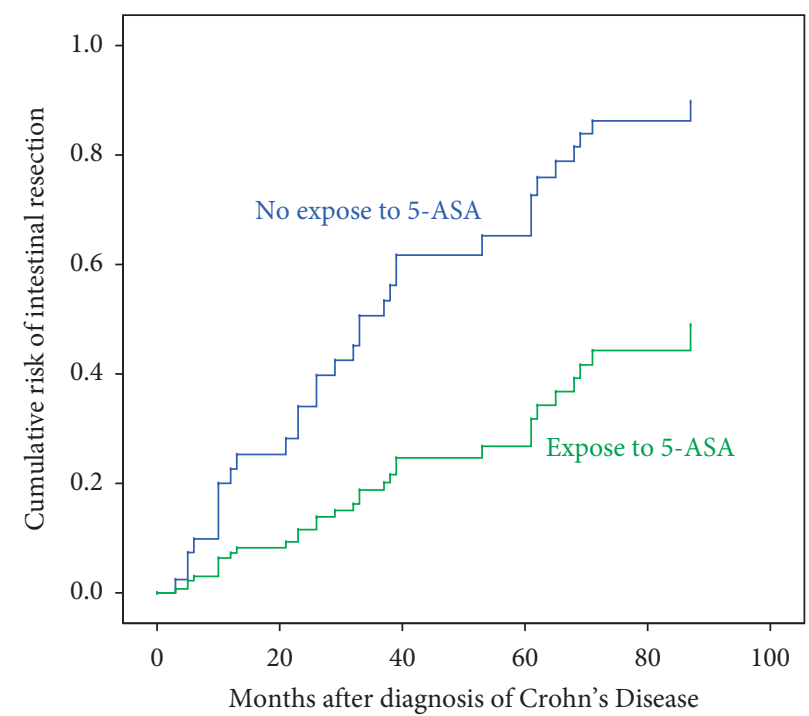

Figure 3: Cumulative risk of change from $B 1$ to $B 2 / B 3$ in patients with $L 2$ and $L 3$ at diagnosis. $B 1$, nonstricturing, nonpenetrating disease; $B 2$, stricturing disease; $B 3$, penetrating disease; $L 2$, colon location; $L 3$, ileocolon location; 5-ASA, 5-aminosalicylic acid.

experienced a change in disease location over time $[4,30]$. Compared with these studies, our study had a higher rate of change in disease location. Disease behavior of CD changed over time $[1,4]$. Results on progression rates varied widely around the world. In a population-based study from Olmsted County, the results showed that the cumulative risk of behavior progression was $33.7 \%$ at 5 years and $50.8 \%$ at 20 years after diagnosis [7]. The cumulative risk of $\mathrm{CD}$ behavior that changed from $\mathrm{B} 1$ to $\mathrm{B} 2 / \mathrm{B} 3$ was $20.4 \%$ in Asia and $16.9 \%$ in Australia in 18 months of follow-up in a population-based study [28]. The progression rate of disease behavior was $42.5 \%$ with a 4.3 years' follow-up in our study, which was higher than the previous populationbased studies. The disease location and behavior progression rates in our study were higher than those in the previous studies. The reason might be that our study was a tertiary hospital-based study while the previous studies were population-based studies, which led to an overrepresentation of severe cases. The patients included in our study might have had more serious disease situation. The progression rate might not represent the real rate of disease behavior progression in China. 
TABLE 3: Factors associated with intestinal resection.

\begin{tabular}{|c|c|c|c|c|c|c|c|c|}
\hline & \multicolumn{4}{|c|}{ All patients $(n=122)$} & \multicolumn{4}{|c|}{$(L 2+L 3)$ patients $(n=98)$} \\
\hline & \multicolumn{2}{|r|}{ Univariate } & \multicolumn{2}{|c|}{ Multivariate } & \multicolumn{2}{|r|}{ Univariate } & \multicolumn{2}{|c|}{ Multivariate } \\
\hline & $P$ & HR $(95 \% \mathrm{CI})$ & $P$ & HR $(95 \% \mathrm{CI})$ & $P$ & HR $(95 \% \mathrm{CI})$ & $P$ & $\mathrm{HR}(95 \% \mathrm{CI})$ \\
\hline Female & 0.105 & $1.73(0.89-3.36)$ & NS & & 0.170 & $1.75(0.79-3.91)$ & NS & \\
\hline \multicolumn{9}{|l|}{ Smoking } \\
\hline Former vs. never & 0.809 & $1.28(0.17-9.44)$ & NS & & NA & & NA & \\
\hline Current vs. never & 0.658 & $0.83(0.36-1.90)$ & NS & & 0.757 & $1.15(0.47-2.79)$ & NS & \\
\hline \multicolumn{9}{|l|}{ Age at diagnosis } \\
\hline$A 2$ vs. $A 1$ & 0.047 & $0.44(0.20-0.99)$ & 0.301 & $1.70(0.62-4.63)$ & 0.507 & $0.69(0.23-2.09)$ & NS & \\
\hline$A 3$ vs. $A 1$ & 0.373 & $0.66(0.26-1.65)$ & 0.094 & $2.15(0.87-6.34)$ & 0.663 & $1.33(0.37-4.76)$ & NS & \\
\hline \multicolumn{9}{|l|}{ Location at diagnosis } \\
\hline$L 2$ vs. $L 1$ & 0.594 & $0.81(0.38-1.73)$ & 0.737 & $0.85(0.33-2.18)$ & NA & & NA & \\
\hline$L 3$ vs. $L 1$ & 0.044 & $0.40(0.16-0.98)$ & 0.713 & $0.82(0.28-2.39)$ & NA & & NA & \\
\hline L4 involvement & 0.979 & $1.02(0.31-3.33)$ & NS & & 0.924 & $1.07(0.25-4.58)$ & NS & \\
\hline \multicolumn{9}{|l|}{ Behavior at diagnosis } \\
\hline$B 2$ vs. $B 1$ & 0.362 & $1.44(0.66-3.15)$ & 0.161 & $1.87(0.78-4.48)$ & 0.259 & $1.69(0.68-4.18)$ & 0.402 & $1.49(0.58-3.82)$ \\
\hline$B 3$ vs. $B 1$ & 0.002 & $5.59(1.86-16.71)$ & 0.022 & $4.65(1.24-17.34)$ & 0.035 & $5.03(1.12-22.59)$ & 0.558 & $1.58(0.34-7.32)$ \\
\hline Perianal disease & 0.410 & $0.43(0.06-3.17)$ & NS & & 0.597 & $0.58(0.08-4.32)$ & NS & \\
\hline Appendicectomy & 0.654 & $1.20(0.55-2.63)$ & NS & & 0.154 & $1.91(0.78-4.64)$ & NS & \\
\hline Elevated ESR at diagnosis & 0.896 & $0.96(0.49-1.87)$ & NS & & 0.538 & $1.30(0.57-2.98)$ & NS & \\
\hline CDAI at diagnosis & 0.420 & $1.00(1.00-1.01)$ & NS & & 0.142 & $1.00(1.00-1.01)$ & NS & \\
\hline 5-Aminosalicylic acid & $\leq 0.001$ & $0.10(0.04-0.23)$ & 0.024 & $0.40(0.20-0.87)$ & $\leq 0.001$ & $0.05(0.01-0.17)$ & 0.027 & $0.33(0.17-0.90)$ \\
\hline Corticosteroid & $\leq 0.001$ & $0.13(0.05-0.33)$ & 0.130 & $0.42(0.14-1.29)$ & 0.001 & $0.15(0.05-0.45)$ & 0.520 & $0.66(0.18-2.37)$ \\
\hline Immunomodulators & $\leq 0.001$ & $0.11(0.04-0.28)$ & 0.030 & $0.29(0.09-0.88)$ & $\leq 0.001$ & $0.06(0.01-0.26)$ & 0.089 & $0.22(0.04-1.26)$ \\
\hline Biologics & 0.001 & $0.25(0.11-0.57)$ & 0.082 & $0.45(0.18-1.11)$ & 0.001 & $0.13(0.04-0.45)$ & 0.023 & $0.24(0.07-0.82)$ \\
\hline
\end{tabular}

HR, hazard ratio; ESR, erythrocyte sedimentation rate; NA, not available; NS, bot significance $(P>0.10)$ in the univariate Cox regression; CDAI: Crohn's disease activity index.

The use of 5-ASA in CD is not well established. Previous studies mainly focused on the efficacy of 5-ASA in the remission induction and maintenance of $C D$ patients [19]. It is contradictory whether oral 5-ASA was effective compared with placebo for induction and maintenance of remission in CD patients [3]. Although the ECCO consensus recommended against the use of 5ASA in CD [10], several meta-analyses showed that only sulfasalazine had modest effect on induction of remission $[31,32]$ and 5-ASA might reduce the probability of clinical relapse in surgically induced remission CD patients $[33,34]$. In the Swiss IBD cohort study, surveyed physicians judged response to 5-ASA treatment as clinically successful in $46 \%$ of treatment episodes [13]. Mesalamine was effective at $4 \mathrm{~g} /$ day as a monotherapy in treatment of active $C D$ [35] and pentasa reduced the CDAI score in comparison with placebo [36]. As colonic $\mathrm{CD}$ patients had different genetics microbiota and serology compared with $\mathrm{CD}$ patients with small intestinal involvement [23], the treatment might be different. Only a few studies reported data separately for colonic CD patients. Sulfasalazine was effective in remission induction in colonic CD patients in two trials [21, 22], but only small samples of colonic CD patients were included in these two studies. Our study included a large number of $L 2$ and $L 3$ patients and focused on the effectiveness in the long-term outcomes, such as disease behavior progression and surgery, instead of remission induction and maintenance. Interestingly, the use of 5-ASA was a protective factor associated with disease behavior progression and intestinal resection in patients presented with $L 2$ and $L 3$ at diagnosis. But patients who presented with $L 1$ at diagnosis did not have the same protective effects when taking 5-ASA. 5-ASA was still widely used in mild CD patients in China [37]. These results emphasized the importance of use 5-ASA in the daily management of colonic involved CD. Early use of 5-ASA may be useful to reduce the rate of disease behavior progression and the surgery in colon involved CD patients. The use of 5-ASA might have some good effects on the long-term outcome of colon involved patients. Large sample of randomized controlled trial was needed to confirm our study result in the colon involved patients.

There were some strengths of the present study. First, our study focused on the efficacy of 5-ASA in the long-term outcome of CD patients. Second, as the efficacy of treatment might be different in CD patients with different disease location, subgroup analysis was performed based on the disease location.

There were also several limitations in our study. First, patients were from a single-center hospital in China. Second, due to the retrospective nature of our study, some potential factors, such as c-reactive protein, endoscopic severity, and histological inflammation score, were not included in our study. In the future, large-sample population-based prospective long-term follow-up studies are needed to confirm our conclusions.

\section{Conclusions}

In conclusion, the use of 5-ASA could reduce the risk of disease behavior progression and intestinal resection in 
colonic and ileocolonic CD patients. The result emphasized the importance of 5-ASA use in preventing disease behavior progression and intestinal resection in $\mathrm{CD}$ patients with colon involvement.

\section{Data Availability}

The datasets used and/or analyzed during the current study are available from the corresponding author upon reasonable request.

\section{Conflicts of Interest}

The authors declare that they have no conflicts of interest.

\section{Authors' Contributions}

K.W., J.L., and J.W. designed the study. J.W., X.W., Y.Z., X.X., Y.L., Z.L., S.H., M.C., and Y.N. collected the data. J.W. and X.W. analyzed the data. X.W. and K.W. revised the statistical analyses. J.W. and X.W. wrote the paper. X.W., Y.Z., Y.S., J.L., and K.W. revised the paper. All the authors approved the final version of the manuscript. Guarantor of the article is Kaichun Wu. Jian Wan, Xuan Wang, and Yujie Zhang contributed equally to this work.

\section{Acknowledgments}

Our study was supported by National Natural Science Foundation of China (81421003, 81627807, 81772650, 81322037, and 81572302), National Key Research and Development Plan (2017YFC0908300), and Independent Funds of the Key Laboratory (CBSKL2015Z01).

\section{Supplementary Materials}

Table S1. Factors associated with change in location in both $L 1$ and $L 2$ patients $(n=74)$. Table S2. Factors associated with intestinal resection in $L 1$ patients $(n=24)$. (Supplementary Materials)

\section{References}

[1] J. Torres, S. Mehandru, J.-F. Colombel, and L. Peyrin-Biroulet, "Crohn's disease," The Lancet, vol. 389, no. 10080, pp. 1741-1755, 2017.

[2] A. N. Ananthakrishnan, "Epidemiology and risk factors for IBD," Nature Reviews Gastroenterology \& Hepatology, vol. 12, no. 4, pp. 205-217, 2015.

[3] G. R. Lichtenstein, E. V. Loftus, K. L. Isaacs, M. D. Regueiro, L. B. Gerson, and B. E. Sands, "ACG clinical guideline: Management of Crohn's disease in adults," American Journal of Gastroenterology, vol. 113, no. 4, pp. 481-517, 2018.

[4] L. Peyrin-Biroulet, E. V. Loftus Jr., J.-F. Colombel, and W. J. Sandborn, "The natural history of adult Crohn's disease in population-based cohorts," American Journal of Gastroenterology, vol. 105, no. 2, pp. 289-297, 2010.

[5] Y. Li, B. Chen, X Gao et al., "Current diagnosis and management of Crohn's disease in China: Results from a multicenter prospective disease registry," BMC Gastroenterology, vol. 19, p. 145, 2019.
[6] B. Lo, M. K. Vester-Andersen, I. Vind et al., "Changes in disease behaviour and location in patients with Crohn's disease after seven years of follow-up: A Danish populationbased inception cohort," Journal of Crohn's and Colitis, vol. 12, no. 3, pp. 265-272, 2018.

[7] K. T. Thia, W. J. Sandborn, W. S. Harmsen, A. R. Zinsmeister, and E. V. Loftus, "Risk factors associated with progression to intestinal complications of Crohn's disease in a populationbased cohort," Gastroenterology, vol. 139, no. 4, pp. 1147-1155, 2010.

[8] D. Duricova, M. Fumery, V. Annese, P. L. Lakatos, L. PeyrinBiroulet, and C. Gower-Rousseau, "The natural history of Crohn's disease in children," European Journal of Gastroenterology \& Hepatology, vol. 29, no. 2, pp. 125-134, 2017.

[9] J. Torres, F. Caprioli, K. H. Katsanos et al., "Predicting outcomes to optimize disease management in inflammatory bowel diseases," Journal of Crohn's and Colitis, vol. 10, no. 12, pp. 1385-1394, 2016.

[10] J. Torres, S. Bonovas, G Doherty et al., "ECCO guidelines on therapeutics in Crohn's disease: Medical treatment," Journal of Crohn's \& colitis, vol. 14, pp. 4-22, 2020.

[11] R. B. Gearry, Y. Ajlouni, S. Nandurkar, J. H. Iser, and P. R. Gibson, "5-Aminosalicylic acid (mesalazine) use in Crohn's disease: A survey of the opinions and practice of Australian gastroenterologists," Inflammatory Bowel Diseases, vol. 13, no. 8, pp. 1009-1015, 2007.

[12] T. Klag, E. F. Stange, and J. Wehkamp, "Management of Crohn's disease-are guidelines transferred to clinical practice?" United European Gastroenterology Journal, vol. 3, no. 4, pp. 371-380, 2015.

[13] A. M. Schoepfer, M. Bortolotti, V. Pittet et al., "The gap between scientific evidence and clinical practice: 5-aminosalicylates are frequently used for the treatment of Crohn's disease," Alimentary Pharmacology \& Therapeutics, vol. 40, no. 8, pp. 930-937, 2014.

[14] C. Sokollik, N. Fournier, D. Rizzuti et al., "The use of 5aminosalicylic acid in children and adolescents with inflammatory bowel disease," Journal of Clinical Gastroenterology, vol. 52, no. 10, pp. e87-e91, 2018.

[15] A. C. I. W. Group, "Retrospective analysis of 515 cases of Crohn's disease hospitalization in China: nationwide study from 1990 to 2003," Journal of Gastroenterology and Hepatology, vol. 21, no. 6, pp. 1009-1015, 2006.

[16] L. Jiang, B. Xia, J. Li et al., "Retrospective survey of 452 patients with inflammatory bowel disease in Wuhan city, central China," Inflammatory Bowel Diseases, vol. 12, no. 3, pp. 212-217, 2006.

[17] X.-m. Song, X. Gao, M.-z. Li et al., "Clinical features and risk factors for primary surgery in 205 patients with Crohn's disease: analysis of a South China cohort," Diseases of the Colon \& Rectum, vol. 54, no. 9, pp. 1147-1154, 2011.

[18] H. Zhai, A. Liu, W. Huang et al., "Increasing rate of inflammatory bowel disease: A 12-year retrospective study in NingXia, China," BMC Gastroenterology, vol. 16, no. 1, p. 2, 2016.

[19] A. C. Ford, S. V. Kane, K. J. Khan et al., "Efficacy of 5aminosalicylates in Crohn's disease: systematic review and meta-analysis," American Journal of Gastroenterology, vol. 106, no. 4, pp. 617-629, 2011.

[20] C. Ma, S. J. Dutton, L. E. Cipriano et al., "Systematic review with meta-analysis: Prevalence, risk factors and costs of aminosalicylate use in Crohn's disease," Alimentary Pharmacology \& Therapeutics, vol. 48, no. 2, pp. 114-126, 2018. 
[21] H. Malchow, K. Ewe, J. W. Brandes et al., "European cooperative Crohn's disease study (ECCDS): Results of drug treatment," Gastroenterology, vol. 86, no. 2, pp. 249-266, 1984.

[22] R. W. Summers, D. M. Switz, J. T. Sessions et al., "National cooperative Crohn's disease study: Results of drug treatment," Gastroenterology, vol. 77, no. 4, pp. 847-869, 1979.

[23] S. Subramanian, A. Ekbom, and J. M. Rhodes, "Recent advances in clinical practice: a systematic review of isolated colonic Crohn's disease: The third IBD?" Gut, vol. 66, no. 2, pp. 362-381, 2017.

[24] G. Van Assche, A. Dignass, J. Panes et al., "The second European evidence-based consensus on the diagnosis and management of Crohn's disease: Definitions and diagnosis," Journal of Crohn's and Colitis, vol. 4, no. 1, pp. 7-27, 2010.

[25] F. Gomollón, A. Dignass, V. Annese et al., "3rd European evidence-based consensus on the diagnosis and management of Crohn's disease 2016: Part 1: Diagnosis and medical management," Journal of Crohn's and Colitis, vol. 11, no. 1, pp. 3-25, 2017.

[26] M. S. Silverberg, J. Satsangi, T. Ahmad et al., "Toward an integrated clinical, molecular and serological classification of inflammatory bowel disease: Report of a working party of the 2005 Montreal world congress of gastroenterology," Canadian Journal of Gastroenterology, vol. 19, no. Suppl A, pp. 5A-36A, 2005.

[27] F. L. Wolters, M. G. Russel, J Sijbrandij et al., "Phenotype at diagnosis predicts recurrence rates in Crohn's disease," Gut, vol. 55, pp. 1124-1130, 2006.

[28] S. C. Ng, Z. Zeng, O. Niewiadomski et al., "Early course of inflammatory bowel disease in a population-based inception cohort study from 8 countries in Asia and Australia," Gastroenterology, vol. 150, no. 1, pp. 86-95, 2016.

[29] E. Louis, A. Collard, A. F. Oger et al., "Behaviour of Crohn's disease according to the Vienna classification: changing pattern over the course of the disease," Gut, vol. 49, no. 6 , pp. 777-782, 2001.

[30] M. Henriksen, J. Jahnsen, I. Lygren et al., "Clinical course in Crohn's disease: Results of a five-year population-based follow-up study (the IBSEN study)," Scandinavian Journal of Gastroenterology, vol. 42, no. 5, pp. 602-610, 2007.

[31] W. C. Lim, Y. Wang, J. K. MacDonald, and S Hanauer, "Aminosalicylates for induction of remission or response in Crohn's disease," Cochrane Database of Systematic Reviews, vol. 7, Article ID CD008870, 2016.

[32] A. K. Akobeng, D. Zhang, M. Gordon, and J. K MacDonald, "Oral 5-aminosalicylic acid for maintenance of medicallyinduced remission in Crohn's disease," Cochrane Database of Systematic Reviews, vol. 9, Article ID CD003715, 2016.

[33] Z. Iheozor-Ejiofor, M. Gordon, A. Clegg et al., "Interventions for maintenance of surgically induced remission in Crohn's disease: a network meta-analysis," Cochrane Database of Systematic Reviews, vol. 9, Article ID CD013210, 2019.

[34] A. C. Ford, K. J. Khan, N. J. Talley, and P. Moayyedi, "5aminosalicylates prevent relapse of Crohn's disease after surgically induced remission: Systematic review and metaanalysis," American Journal of Gastroenterology, vol. 106, no. 3, pp. 413-420, 2011.

[35] J. W. Singleton, S. B. Hanauer, G. L. Gitnick et al., "Mesalamine capsules for the treatment of active Crohn's disease: Results of a 16-week trial," Gastroenterology, vol. 104, no. 5, pp. 1293-1301, 1993.

[36] S. Hanauer and U. Stromberg, "Oral pentasa in the treatment of active Crohn's disease: A meta-analysis of double-blind, placebo-controlled trials," Clinical Gastroenterology and Hepatology, vol. 2, no. 5, pp. 379-388, 2004.

[37] M. Chen, F. Yi, F. Zhou et al., "Risk factors for initial surgery in patients with Crohn's disease in Central China," Surgery Today, vol. 45, no. 8, pp. 1002-1008, 2015. 